\title{
DUAL OF 2D FRACTIONAL FOURIER TRANSFORM ASSOCIATED TO ITÔ-HERMITE POLYNOMIALS
}

\author{
ABDELHADI BENAHMADI AND ALLAL GHANMI
}

Dedicated to the memory of Professor Elhachmia Ait Benhaddou

\begin{abstract}
A class of integral transforms, on the planar Gaussian Hilbert space with range in the weighted Bergman space on the bi-disk, is defined as the dual transforms of the $2 \mathrm{~d}$ fractional Fourier transform associated with the Mehler function for ItôHermite polynomials. Some spectral properties of these transforms are investigated. Namely, we study their boundedness and identify their null spaces as well as their ranges. Such identification depends on the zeros set of Itô-Hermite polynomials. Moreover, the explicit expressions of their singular values are given and compactness and membership in $p$-Schatten class are studied. The relationship to specific fractional Hankel transforms is also established
\end{abstract}

\section{INTRODUCTION}

The role played by the classical Mehler formula [14,

$$
\sum_{n=0}^{+\infty} \frac{t^{n} H_{n}(x) H_{n}(y)}{2^{n} n !}=\frac{1}{\sqrt{1-t^{2}}} \exp \left(\frac{-t^{2}\left(x^{2}+y^{2}\right)+2 t x y}{1-t^{2}}\right),
$$

for the real Hermite polynomials $H_{n}(x):=(-1)^{n} e^{x^{2}} \frac{d^{n}}{d x^{n}}\left(e^{-x^{2}}\right)$, is well known in the literature [14, 18, 3, 12, 15, 17, 13, 8, 16]. Its complex analogues for Itô-Hermite polynomials $H_{m, n}^{\nu}$ have been obtained in [19, 4, 10, 6] and have been employed in [6] to establish integral reproducing property for $H_{m, n}^{\nu}$ by a like Fourier transform, and to provide a closed expression of the heat kernel for the Cauchy initial value problem for a special magnetic Laplacian acting on the Hilbert space $L_{\nu}^{2}(\mathbb{C}):=L^{2, \nu}\left(\mathbb{C} ; e^{-\nu|z|^{2}} d \lambda\right)$. In [20], Zayed has used the one in (2.1) below to construct a non trivial $2 \mathrm{~d}$ fractional Fourier transform

$$
\mathcal{F}_{u, v}^{\nu} \psi(\xi)=\int_{\mathbb{C}} \psi(\zeta) K_{u, v}^{\nu}(\zeta ; \xi) e^{-\nu|\zeta|^{2}} d x d y ; \zeta=x+i y,
$$

whose eigenfunctions are the Itô-Hermite polynomials. Here $K_{u, v}^{\nu}(\zeta ; \xi)$ stands for the kernel function

$$
K_{u, v}^{\nu}(\zeta ; \xi)=\frac{\nu}{\pi(1-u v)} \exp \left(\frac{\nu}{1-u v}\left\{-u v\left(|\zeta|^{2}+|\xi|^{2}\right)+u \bar{\zeta} \xi+v \zeta \bar{\xi}\right\}\right) .
$$

In the present paper, we explore further applications in the context of integral transforms and weighted Bergman spaces $B_{\alpha, \beta}^{2}\left(D^{2}\right)$ on the bi-disk $D^{2}=D \times D$; $D=\{z \in \mathbb{C}, z \bar{z}<1\}$, defined as the Hilbert space of all analytic functions on $D^{2}$ that are square integrable with respect to the measure

$$
d \mu_{\alpha, \beta}(z, w)=\omega_{\alpha, \beta}\left(|z|^{2},|w|^{2}\right) d \lambda(z, w),
$$

2010 Mathematics Subject Classification. Primary 44A20; 30G35; 30H20 Secondary 47B38; 30D55. Key words and phrases. Weighted Bergman space on bi-disk; Itô-Hermite polynomials; Mehler formula; Singular values; $p$-Schatten class; Segal-Bargmann transform. 
where the weight function is given by

$$
\omega_{\alpha, \beta}(s, t):=(1-s)^{\alpha}(1-t)^{\beta} ; \alpha, \beta>-1,
$$

and $d \lambda$ denotes the standard Lebesgue measure. To this end we follow the scheme already applied in [7] to introduce and study the dual transforms of fractional Hankel transforms with ranges in weighted Bergman space on the disk. Mainly, we consider the family of integral transforms

$$
\mathcal{R}_{w}^{\nu} f(u, v)=\int_{\mathbb{C}} f(z) K_{u, v}^{\nu}(z ; w) e^{-\nu|z|^{2}} d \lambda(z)
$$

on $D^{2}$, labeled by $\nu>0$ and $w \in \mathbb{C}$ and seen as the dual transform of the $2 \mathrm{~d}$ fractional Fourier transform in $(1.2), \mathcal{R}_{w}^{\nu} f(u, v)=\mathcal{F}_{u, v}^{\nu} f(w)$.

The aim in this paper concern identification of the null space and the range of the transforms $\mathcal{R}_{w}^{\nu}$. We also study their boundedness and provide complete description of their compactness and membership in $p$-Schatten class. Our main results can be stated as follows

Theorem 1.1. The integral transform $\mathcal{R}_{w}^{\nu}$ is well defined and bounded from $L_{\nu}^{2}(\mathbb{C})$ into the weighted Bergman space $B_{\alpha, \beta}^{2}\left(D^{2}\right)$ if and only if $\alpha>0$ and $\beta>0$. The characterization of its null space $\operatorname{Ker}\left(\mathcal{R}_{w}^{\nu}\right)$ depends on the zeros set of Itô-Hermite polynomials $H_{m, n}^{\nu}$. Namely, if $N_{w}(H)=\left\{(m, n) ; m, n=0,1,2, \cdots ; H_{m, n}^{\nu}(w, \bar{w})=0\right\}$, then $\operatorname{Ker}\left(\mathcal{R}_{w}^{\nu}\right)$ is a vector space spanned as

$$
\operatorname{Ker}\left(\mathcal{R}_{w}^{\nu}\right)=\operatorname{Span}\left\{H_{m, n}^{\nu} ;(m, n) \in N_{w}(H)\right\} .
$$

Theorem 1.2. For $\alpha, \beta>0$, the operator $\mathcal{R}_{w}^{\nu}: L_{\nu}^{2}(\mathbb{C}) \longrightarrow B_{\alpha, \beta}^{2}\left(D^{2}\right)$ is compact and its singular values are given by

$$
s_{m, n}^{\nu, \alpha, \beta}(w)=\left(\frac{\nu \pi \Gamma(\alpha+1) \Gamma(\beta+1)}{\nu^{m+n} \Gamma(\alpha+m+2) \Gamma(\beta+n+2)}\right)^{1 / 2}\left|H_{m, n}^{\nu}(w, \bar{w})\right| .
$$

Moreover, it belongs to the $p$-Schatten class for every $p>\max (2 /(\alpha+1) ; 2 /(\beta+1))$.

The proof of Theorem 1.1 is contained in Propositions 3.1, 3.2, 3.3 and 3.5 presented in Section 3, while the one of Theorem 1.2 is given in Section 4. The next section is devoted to some preliminaries concerning weighted Bergman space on the bi-disk and Itô-Hermite polynomials. We conclude the paper by discussing the close connection of $\mathcal{R}_{w}^{\nu}$ to the fractional Hankel transforms

$$
\mathcal{H}_{u, v}^{\nu, \alpha}(f)(y)=\frac{2 \nu}{1-u v}\left(\frac{u}{v}\right)^{\alpha / 2} \int_{0}^{\infty} x f(x) I_{\alpha}\left(\frac{2 \nu \sqrt{u v}}{1-u v} x y\right) e^{\frac{-\nu\left(x^{2}+u v y^{2}\right)}{1-u v}} d r
$$

where $I_{\alpha}$ denotes the modified Bessel function [1, p.222].

Theorem 1.3. Let $f \in L_{\nu}^{2}(\mathbb{C})$ and $g_{k}$ the associated Fourier coefficients. Then, $\mathcal{H}_{u, v}^{\nu, \alpha}\left(g_{k}\right)$, for varying integer $k$, are the fractional Fourier coefficients of $\mathcal{F}_{u, v}^{\nu} f$, the fractional Fourier transform of $f$.

\section{PRELIMINARIES}

For fixed reals $\alpha, \beta>-1$, the weighted Bergman space $B_{\alpha, \beta}^{2}\left(D^{2}\right)$ is a closed subspace of the Hilbert space $L_{\alpha, \beta}^{2}\left(D^{2}\right):=L^{2}\left(\mathbb{D} \times \mathbb{D} ; d \mu_{\alpha, \beta}\right)$ endowed with the scaler product

$$
\langle f, g\rangle_{\alpha, \beta}=\int_{D^{2}} f(z, w) \overline{g(z, w)} d \mu_{\alpha, \beta}(z, w) .
$$


We denote by $\|\cdot\|_{\alpha, \beta}$ the associated norm. An orthonormal basis of $B_{\alpha, \beta}^{2}\left(D^{2}\right)$ is given by

$$
\varphi_{m, n}^{\alpha, \beta}:=\left(\gamma_{m, n}^{\alpha, \beta}\right)^{-1 / 2} e_{m, n}
$$

where $e_{m, n}(z, w):=z^{m} w^{n}$ and $\gamma_{m, n}^{\alpha, \beta}$ is its square norm given by

$$
\gamma_{m, n}^{\alpha, \beta}:=\frac{\pi^{2} \Gamma(\alpha+1) \Gamma(\beta+1) m ! n !}{\Gamma(\alpha+m+2) \Gamma(\beta+n+2)}=\left\|e_{m, n}\right\|_{\alpha, \beta}^{2} .
$$

Subsequently, the sequential characterization of $B_{\alpha, \beta}^{2}\left(D^{2}\right)$ is given by

$$
B_{\alpha, \beta}^{2}\left(D^{2}\right)=\left\{\sum_{m, n=0}^{\infty} a_{m ; n} e_{m, n} ; \sum_{m, n=0}^{\infty} \gamma_{m, n}^{\alpha, \beta}\left|a_{m ; n}\right|^{2}<\infty\right\} .
$$

Accordingly, $B_{0,0}^{2}\left(D^{2}\right)$ is identified to be the Hardy space on the bi-disk, while $B_{-1,-1}^{2}\left(D^{2}\right)$ is the classical Bergman space on $D^{2}$. The reproducing kernel of $B_{\alpha, \beta}^{2}\left(D^{2}\right)$ is given by

$$
K_{\alpha, \beta}((u, v) ;(z, w))=\frac{(\alpha+1)(\beta+1)}{\pi^{2}(1-u \bar{z})^{\alpha+2}(1-v \bar{w})^{\beta+2}} .
$$

Thus $B_{\alpha, \beta}^{2}\left(D^{2}\right)$ is obtained as the Bergman projection

$$
P(\varphi)(u, v)=\frac{(\alpha+1)(\beta+1)}{\pi^{2}} \int_{D^{2}} \frac{\varphi(z, w)}{(1-u \bar{z})^{\alpha+2}(1-v \bar{w})^{\beta+2}} d \mu_{\alpha, \beta}(z, w)
$$

of $L_{\alpha, \beta}^{2}\left(D^{2}\right)$. Another realization is by means of the unitary two-dimensional second Bargmann transform

$$
\mathcal{B}_{\alpha, \beta} \varphi(z, w)=\frac{1}{(1-z)^{\alpha+1}(1-w)^{\beta+1}} \int_{\mathbb{R}^{+2}} s^{\alpha} t^{\beta} \exp \left(\frac{s w+t z-(s+t)}{(1-z)(1-w)}\right) \varphi(s, t) d s d t
$$

acting on the Hilbert space $L^{2}\left(\mathbb{R}^{+2} ; x^{\alpha} y^{\beta} e^{-x-y} d x d y\right)$. The kernel function of $\mathcal{B}_{\alpha, \beta}$ appears as the tensor product of two copies of the kernel function of the standard onedimensional second Bargmann transform [2, p. 203]. However, it can be seen as the bilinear generating function involving the product of generalized Laguerre polynomials $L_{m}^{(\alpha)}(s) L_{n}^{(\beta)}(t)$.

In the sequel, we will provide interesting realization of specific subspaces of $B_{\alpha, \beta}^{2}\left(D^{2}\right)$ by invoking the complex Mehler function

$$
K_{u, v}^{\nu ; \nu^{\prime}}(z, w)=\frac{1}{1-u v} \exp \left(\frac{-u v\left(\nu|z|^{2}+\nu^{\prime}|w|^{2}\right)+\nu^{\prime} u z w+\nu v \overline{z w}}{1-u v}\right)
$$

with $\nu, \nu^{\prime}>0$ and $u, v \in D$, associated to Itô-Hermite polynomials $H_{m, n}^{\nu}$ defined on the complex plane $\mathbb{C}$ by [11, 9, [5]

$$
H_{m, n}^{\nu}(z, \bar{z})=(-1)^{m+n} e^{\nu z \bar{z}} \frac{\partial^{m+n}}{\partial \bar{z}^{m} \partial z^{n}}\left(e^{-\nu z \bar{z}}\right)
$$

To this end, let recall that the $H_{m, n}^{\nu}$ form an orthogonal basis of $L_{\nu}^{2}(\mathbb{C})$ and that the Mehler function in 2.1 can be expanded in terms of normalized Itô-Hermite polynomials

as [6]

$$
\psi_{m, n}^{\nu}:=\left(\frac{\nu}{\pi \nu^{m+n} m ! n !}\right)^{1 / 2} H_{m, n}^{\nu^{\prime}}
$$

$$
K_{u, v}^{\nu ; \nu^{\prime}}(z, w):=\sum_{m, n=0}^{\infty} u^{m} v^{n} \psi_{m, n}^{\nu}(z) \psi_{m, n}^{\nu}(w)
$$


For $\nu=\nu^{\prime}=1$, this is exactly the one announced by Wünsche [19] and proved later by Ismail in [10, Theorem 3.3] as a specific case of his Kibble-Slepian formula [10, Theorem $1.1]$.

\section{Basic PROPERTIES OF $\mathcal{R}_{w}^{\nu}$}

We begin by observing that the kernel function in 1.3 reads in terms of the one in (2.1) as

$$
K_{u, v}^{\nu}(z, w)=\left(\frac{\nu}{\pi}\right) K_{u, v}^{\nu ; \nu}(\bar{z}, w)
$$

and therefore satisfies

$$
\int_{\mathbb{C}} K_{u, v}^{\nu}(z ; w) \overline{K_{u, v}^{\nu}(z ; w)} e^{-\nu|z|^{2}} d \lambda(z)=K_{|u|^{2},|v|^{2}}^{\nu}(w ; w)>0 .
$$

Proposition 3.1. The integral transform $\mathcal{R}_{w}^{\nu}$ is well defined on $L_{\nu}^{2}(\mathbb{C})$.

Proof. Using (3.2) and the Cauchy-Schwarz inequality, we obtain

$$
\begin{aligned}
\left|\mathcal{R}_{w}^{\nu} f(u, v)\right| & \leq\left(\int_{\mathbb{C}}\left|K_{u, v}^{\nu}(w ; w)\right|^{2}\right)^{1 / 2}\|f\|_{L_{\nu}^{2}(\mathbb{C})} \\
& \leq\left(K_{|u|^{2},|v|^{2}}^{\nu}(w ; w)\right)^{1 / 2}\|f\|_{L_{\nu}^{2}(\mathbb{C})}
\end{aligned}
$$

for every $f \in L_{\nu}^{2}(\mathbb{C})$.

The action of $\mathcal{R}_{w}^{\nu}$ on $\psi_{m, n}^{\nu}$ in 2.3 is given by

$$
\mathcal{R}_{w}^{\nu} \psi_{m, n}^{\nu}=\psi_{m, n}^{\nu}(w) e_{m, n}
$$

This follows by means of (3.1) and 2.4). Therefore, the family $\mathcal{R}_{w}^{\nu} \psi_{m, n}^{\nu}$, for varying $m, n$, form an orthogonal system in $L_{\alpha, \beta}^{2}\left(D^{2}\right)$ since the monomials $e_{m, n}$ are. The next result discusses the boundedness of $\mathcal{R}_{w}^{\nu}$ from $L_{\nu}^{2}(\mathbb{C})$ into the weighted Hilbert space $L_{\alpha, \beta}^{2}\left(D^{2}\right)$.

Proposition 3.2. For $\alpha>0$ and $\beta>0$, the operator $\mathcal{R}_{w}^{\nu}$ is bounded from $L_{\nu}^{2}(\mathbb{C})$ into $L_{\alpha, \beta}^{2}\left(D^{2}\right)$.

Proof. Set

$$
k_{w}^{\nu, \alpha, \beta}:=\int_{D^{2}} K_{|u|^{2},|v|^{2}}^{\nu}(w ; w) d \mu_{\alpha, \beta}(u, v) .
$$

Then, from (3.3), we have

$$
\left\|\mathcal{R}_{w}^{\nu} f\right\|_{\alpha, \beta}^{2} \leq k_{w}^{\nu, \alpha, \beta}\|f\|_{L_{\nu}^{2}(\mathbb{C})}^{2} .
$$

Subsequently, the boundedness of the operator $\mathcal{R}_{w}^{\nu}$ requires that $k_{w}^{\nu, \alpha, \beta}$ be finite. But, using the closed expression of $K_{|u|^{2},|v|^{2}}^{\nu}(w ; w)$, we get

$$
k_{w}^{\nu, \alpha, \beta}=\nu \pi \int_{0}^{1} \int_{0}^{1} \exp \left(\frac{\nu(s+t-2 s t)}{1-s t}|w|^{2}\right) \frac{\omega_{\alpha, \beta}(s, t)}{1-s t} d s d t .
$$

Hence since $0 \leq(s+t-2 s t) /(1-s t) \leq 1$ and $1 /(1-s t) \leq 1 /(1-s)(1-t)$, it follows

$$
\nu \pi \int_{0}^{1} \int_{0}^{1} \omega_{\alpha, \beta}(s, t) d s d t \leq k_{w}^{\nu, \alpha, \beta} \leq \nu \pi e^{\nu|w|^{2}} \int_{0}^{1} \int_{0}^{1} \omega_{\alpha-1, \beta-1}(s, t) d s d t .
$$

Thus, $\mathcal{R}_{w}^{\nu}$ is bounded for $\alpha, \beta>0$. In this case $\mathcal{R}_{w}^{\nu} f$ belongs to $L_{\alpha, \beta}^{2}\left(D^{2}\right)$ for every $f \in L_{\nu}^{2}(\mathbb{C})$. 
Now, appealing to the fact that $\psi_{m, n}^{\nu}$ constitutes an orthonormal basis of $L_{\nu}^{2}(\mathbb{C})$, we can expand any $f \in L_{\nu}^{2}(\mathbb{C})$ as $f=\sum_{m, n=0}^{\infty} \alpha_{m, n} \psi_{m, n}^{\nu}$, so that one gets

$$
\mathcal{R}_{w}^{\nu} f=\sum_{m, n=0}^{\infty} \alpha_{m, n} \psi_{m, n}^{\nu}(w)\left(\gamma_{m, n}^{\alpha, \beta}\right)^{1 / 2} \varphi_{m, n}^{\alpha, \beta}
$$

The series in (3.8) converges uniformly on compact sets of the complex plane. Direct computation shows that we have

$$
\left\|\mathcal{R}_{w}^{\nu} f\right\|_{\alpha, \beta}^{2}=\sum_{m, n=0}^{\infty}\left|\alpha_{m, n}\right|^{2}\left|\psi_{m, n}^{\nu}(w)\right|^{2} \gamma_{m, n}^{\alpha, \beta}
$$

Accordingly, the description of the range and the null space of the $\mathcal{R}_{w}^{\nu}$ are closely connected to zeros of Itô-Hermite polynomials. Thus, we let $\mathcal{Z}\left(H_{m, n}^{\nu}\right)$ denotes the zeros set of $H_{m, n}^{\nu}$ for fixed $m, n$, while $\mathcal{Z}(H):=\cup_{m, n} \mathcal{Z}\left(H_{m, n}^{\nu}\right)$. We also set

$$
N_{w}(H)=\left\{(m, n) ; m, n=0,1,2, \cdots ; H_{m, n}^{\nu}(w, \bar{w})=0\right\} .
$$

Proposition 3.3. Let $\alpha>0$ and $\beta>0$. If $w \notin \mathcal{Z}(H)$, then the range of $\mathcal{R}_{w}^{\nu}$ acting on $L_{\nu}^{2}(\mathbb{C})$ is a specific subspace of the weighted Bergman space $B_{\alpha, \beta}^{2}\left(D^{2}\right)$.

Proof. This is immediate by means of $(3.8)$ and Proposition 3.2. Indeed, $\mathcal{R}_{w}^{\nu} f$ belongs to $L_{\alpha, \beta}^{2}\left(D^{2}\right)$ and is clearly holomorphic on $D^{2}$ by Stone-Weierstrass theorem. Hence, $\mathcal{R}_{w}^{\nu}\left(L_{\nu}^{2}(\mathbb{C})\right) \subset B_{\alpha, \beta}^{2}\left(D^{2}\right)$.

Remark 3.4. Concerning the converse inclusion, we can provide an explicit example showing that the range of $L_{\nu}^{2}(\mathbb{C})$ by $\mathcal{R}_{w}^{\nu}$ is strictly contained in $B_{\alpha, \beta}^{2}\left(D^{2}\right)$. However, this can be reproved using compactness (discussed below) of the transform $\mathcal{R}_{w}^{\nu}$, since the range of compact operator is not closed in $B_{\alpha, \beta}^{2}\left(D^{2}\right)$ ay least for in $\alpha, \beta>0$.

Proposition 3.5. The null space of $\mathcal{R}_{w}^{\nu}$ acting on $L_{\nu}^{2}(\mathbb{C})$ is a vector space characterized explicitly as

$$
\operatorname{Ker}\left(\mathcal{R}_{w}^{\nu}\right)=\operatorname{Span}\left\{H_{m, n} ;(m, n) \in N_{w}(H)\right\}
$$

with dimension equals to the cardinal of $N_{w}(H)$. Thus, the integral transform $\mathcal{R}_{w}^{\nu}$ is one-to-one if and only if $w \notin \mathcal{Z}(H)$.

Proof. According to (3.9), if $f=\sum_{m, n=0}^{\infty} \alpha_{m, n} \psi_{m, n}^{\nu} \in L_{\nu}^{2}(\mathbb{C})$ is in the null space of $\mathcal{R}_{w}^{\nu}$, then $\left\|\mathcal{R}_{w}^{\nu} f\right\|=0$ and hence $\alpha_{m, n} \psi_{m, n}^{\nu}(w)=0$ for every $m, n$. Therefore, the null space of $\mathcal{R}_{w}^{\nu}$ reduces to $f=0$ when $w \notin \mathcal{Z}(H)$. Now, for $w \in \mathcal{Z}(H)$, we conclude that $\alpha_{m, n}=0$ for all $(m, n) \notin N_{w}(H)$. Therefore, $f=\sum_{(m, n) \in N_{w}(H)} \alpha_{m, n} \psi_{m, n}^{\nu}$ which proves

$$
\operatorname{Ker}\left(\mathcal{R}_{w}^{\nu}\right) \subset \operatorname{Span}\left\{\psi_{m, n}^{\nu} ;(m, n) \in N_{w}(H)\right\} .
$$

The converse inclusion is trivial and hence $\operatorname{dim}\left(\operatorname{Ker}\left(\mathcal{R}_{w}^{\nu}\right)\right)=\operatorname{Cardinal}(N(H))$.

\section{Proof of Theorem 1.2; Compactness and membership in $p$-Schatten} CLASS

Set

$$
c_{m, n}^{\nu, \alpha, \beta}(w):=\psi_{m, n}^{\nu}(w)\left(\gamma_{m, n}^{\alpha, \beta}\right)^{1 / 2}
$$

and consider the finite rank operators

$$
\mathcal{R}_{p, q} f=\sum_{m=0}^{p} \sum_{n=0}^{q} c_{m, n}^{\nu, \alpha, \beta}(w)\left\langle f, \psi_{m, n}^{\nu}\right\rangle_{L_{\nu}^{2}(\mathbb{C})} \varphi_{m, n}^{\alpha, \beta}
$$


which are bounded and compact. Then, using the fact $\left|c_{m, n}^{\nu, \alpha, \beta}(w)\right|^{2} \leq e^{\nu|w|^{2}} \gamma_{m, n}^{\alpha, \beta}$ as well as $\|f\|_{L_{\nu}^{2}(\mathbb{C})}^{2}=\sum_{m, n=0}^{\infty}\left|\left\langle f, \psi_{m, n}^{\nu}\right\rangle_{L_{\nu}^{2}(\mathbb{C})}\right|^{2}$, we obtain

$$
\begin{aligned}
\left\|\left(\mathcal{R}_{w}^{\nu}-\mathcal{R}_{p, q}\right) f\right\|^{2} & =\sum_{m=p+1}^{\infty} \sum_{n=q+1}^{\infty}\left|c_{m, n}^{\nu, \alpha, \beta}(w)\right|^{2}\left|\left\langle f, \psi_{m, n}^{\nu}\right\rangle_{L_{\nu}^{2}(\mathbb{C})}\right|^{2} \\
& \leq\left(\sum_{m=p+1}^{\infty} \sum_{n=q+1}^{\infty}\left|c_{m, n}^{\nu, \alpha, \beta}(w)\right|^{2}\right)\|f\|_{L_{\nu}^{2}(\mathbb{C})}^{2} \\
& \leq e^{\nu|w|^{2}}\left(\sum_{m=p+1}^{\infty} \sum_{n=q+1}^{\infty} \gamma_{m, n}^{\alpha, \beta}\right)\|f\|_{L_{\nu}^{2}(\mathbb{C})}^{2}
\end{aligned}
$$

so that the following estimate for the operator norm

$$
\left\|\mathcal{R}_{w}^{\nu}-\mathcal{R}_{p, q}\right\|^{2} \leq e^{\nu|w|^{2}} \sum_{m=p+1}^{\infty} \sum_{n=q+1}^{\infty} \gamma_{m, n}^{\alpha, \beta} .
$$

follows. The series in the right-hand side is convergent for $\alpha>0$ and $\beta>0$, and hence its rest goes to zero, so that $\lim _{p, q \longrightarrow \infty}\left\|\mathcal{R}_{p, q}-\mathcal{R}_{w}^{\nu}\right\|=0$.

The above discussion can be reformulated as follows.

Proposition 4.1. Let $\alpha>0$ and $\beta>0$, then $\mathcal{R}_{w}^{\nu}(f)$ is compact.

Remark 4.2. The expansion in (3.8),

$$
\mathcal{R}_{w}^{\nu}(f)=\sum_{m, n=0}^{\infty} c_{m, n}^{\nu, \alpha, \beta}(w)\left\langle f, \psi_{m, n}^{\nu}\right\rangle_{L_{\nu}^{2}(\mathbb{C})} \varphi_{m, n}^{\alpha, \beta},
$$

looks like the spectral decomposition of the operator $\mathcal{R}_{w}^{\nu}$.

From general context, we know that the adjoint of the integral transform $\mathcal{R}_{w}^{\nu}$ is given through

$$
\left(\mathcal{R}_{w}^{\nu}\right)^{*} g(z)=\left\langle g, K_{u, v}^{\nu}(\cdot ; w)\right\rangle_{\alpha, \beta}=\int_{D^{2}} g(u, v) \overline{K_{u, v}^{\nu}(z ; w)} d \mu_{\alpha, \beta}
$$

for every function $g \in L_{\alpha, \beta}^{2}\left(D^{2}\right)$. This can easily be handled by direct computation. Subsequently, $\left(\mathcal{R}_{w}^{\nu}\right)^{*} \mathcal{R}_{w}^{\nu}$ is an integral transform on $L_{\nu}^{2}(\mathbb{C})$

$$
\left(\mathcal{R}_{w}^{\nu}\right)^{*} \mathcal{R}_{w}^{\nu} f=\int_{\mathbb{C}} S_{w}^{\nu}(\zeta, z) f(\zeta) e^{-\nu|\zeta|^{2}} d \lambda(\zeta)
$$

with kernel function given by

$$
S_{w}^{\nu}(\zeta, z):=\sum_{m, n=0}^{\infty}\left|c_{m, n}^{\nu, \alpha, \beta}(w)\right|^{2} \psi_{m, n}^{\nu}(z) \overline{\psi_{m, n}^{\nu}(\zeta)} .
$$

Therefore, we have

$$
\left(\mathcal{R}_{w}^{\nu}\right)^{*} \mathcal{R}_{w}^{\nu}(f)=\sum_{m, n=0}^{\infty}\left|c_{m, n}^{\nu, \alpha, \beta}(w)\right|^{2}\left\langle f, \psi_{m, n}^{\nu}\right\rangle \psi_{m, n}^{\nu}
$$

and in particular

$$
\left(\mathcal{R}_{w}^{\nu}\right)^{*} \mathcal{R}_{w}^{\nu}\left(\psi_{m, n}^{\nu}\right)=\left|c_{m, n}^{\nu, \alpha, \beta}(w)\right|^{2} \psi_{m, n}^{\nu}
$$

Accordingly, since $\psi_{m, n}^{\nu}$ form an orthonormal basis of $L_{\nu}^{2}(\mathbb{C})$, the singular values of $\mathcal{R}_{w}^{\nu}$ which are the eigenvalue of $\left|\mathcal{R}_{w}^{\nu}\right|:=\left(\left(\mathcal{R}_{w}^{\nu}\right)^{*} \mathcal{R}_{w}^{\nu}\right)^{1 / 2}$ are given by

$$
s_{m, n}^{\nu, \alpha, \beta}(w)=\left|c_{m, n}^{\nu, \alpha, \beta}(w)\right|:=\left|\psi_{m, n}^{\nu}(w)\right|\left(\gamma_{m, n}^{\alpha, \beta}\right)^{1 / 2} .
$$


More explicitly,

$$
s_{m, n}^{\nu, \alpha}(w)=\left(\frac{\nu \pi \Gamma(\alpha+1) \Gamma(\beta+1)}{\nu^{m+n} \Gamma(\alpha+m+2) \Gamma(\beta+n+2)}\right)^{1 / 2}\left|H_{m, n}^{\nu}(w, \bar{w})\right| .
$$

Subsequently,

$$
s_{m, n}^{\nu, \alpha, \beta}(w) \leq \pi e^{\frac{\nu|w|^{2}}{2}}\left(\frac{m ! n ! \Gamma(\alpha+1) \Gamma(\beta+1)}{\Gamma(m+\alpha+2) \Gamma(n+\beta+2)}\right)^{\frac{1}{2}} .
$$

It follows that $\lim _{m, n \longrightarrow \infty} s_{m, n}^{\nu, \alpha, \beta}(w)=0$ since $\alpha, \beta>0$ and the right hand-side behaves as $m^{-\alpha-1} n^{-\beta-1}$ for $m, n$ large enough. Moreover, $\mathcal{R}_{w}^{\nu}$ is in the $p$-Schatten class if $p(\alpha+1) / 2>1$ and $p(\beta+1) / 2>1$, i.e. such that $p>\max (2 /(\alpha+1) ; 2 /(\beta+1))$. This readily follows by means of

$$
\left(s_{m, n}^{\nu, \alpha, \beta}(w)\right)^{p} \leq \pi^{p} \frac{\left(e^{\nu|w|^{2}} \Gamma(\alpha+1) \Gamma(\beta+1)\right)^{\frac{p}{2}}}{m^{p(\alpha+1) / 2} n^{p(\beta+1) / 2}} .
$$

Thus we have proved the following

Proposition 4.3. Let $\alpha, \beta>0$. The singular values of $\mathcal{R}_{w}^{\nu}$ are given by (4.1). Moreover, $\mathcal{R}_{w}^{\nu}$ is in $p$-Schatten class for every $p$ such that $p>\max (2 /(\alpha+1) ; 2 /(\beta+1))$.

Remark 4.4. $\mathcal{R}_{w}^{\nu}$ is not a trace class operator if $\alpha \leq 1$ or $\beta \leq 1$. However, it is always a Hilbert-Schmidt operator for $\alpha, \beta>0$.

\section{Connection to Generalized Fractional Hankel transform}

The transform $\psi \longmapsto \mathcal{R}_{w}^{\nu} \psi(u, v)$, for fixed $u, v \in D$, seen as function in the variable $w$, is exactly the non trivial $2 \mathrm{~d}$ fractional Fourier transform considered by Zayed in [20], to wit

$$
\mathcal{F}_{u, v}^{\nu} \psi(\xi)=\int_{\mathbb{C}} \psi(\zeta) K_{u, v}^{\nu}(\zeta ; \xi) e^{-\nu|\zeta|^{2}} d \lambda(\zeta)=\mathcal{R}_{\xi}^{\nu} \psi(u, v),
$$

where $K_{u, v}^{\nu}(\zeta ; \xi)$ is as in 1.3$)$. The eigenfunctions of $\mathcal{F}_{u, v}^{\nu}$ are the Itô-Hermite polynomials,

$$
\mathcal{F}_{u, v}^{\nu} \psi_{m, n}^{\nu}=u^{m} v^{n} \psi_{m, n}^{\nu}
$$

The connection of fractional Fourier coefficients of given $f \in L_{\nu}^{2}(\mathbb{C})$ to its fractional Fourier transform $\mathcal{F}_{u, v}^{\nu} f$ is given in Theorem 1.3 by means of the fractional Hankel transform in (1.7) which is a specific generalization of the classical Hankel transform []. To this end, we begin by interpreting $\mathcal{H}_{u, v}^{\nu, \alpha}$ for integer order $\alpha=k$ as the radial part of the $2 \mathrm{~d}$ Fourier transform $\mathcal{F}_{u, v}^{\nu}$ of $k$-rotationally symmetric function.

Proposition 5.1. For every rotational $\psi_{k}(\zeta)=\Psi(|\zeta|) e^{i k \theta}$, we have

$$
\mathcal{F}_{u, v}^{\nu} \psi_{k}(\xi)=\left(\frac{\xi}{\bar{\xi}}\right)^{k / 2} \mathcal{H}_{u, v}^{\nu, \alpha}(\Psi)(|\xi|)
$$

Proof. By expanding the kernel function $K_{u, v}^{\nu}(\zeta ; \xi) e^{-\nu|\zeta|^{2}}$ in power series as

$$
K_{u, v}^{\nu}(\zeta ; \xi) e^{-\nu|\zeta|^{2}}=\exp \left(\frac{-\nu\left(|\zeta|^{2}+u v|\xi|^{2}\right)}{1-u v}\right) \sum_{m, n=0}^{\infty} \frac{\nu^{m+n}(u \xi)^{m}(v \bar{\xi})^{n}}{(1-u v)^{m+n}} \frac{\zeta^{n} \bar{\zeta}^{m}}{m ! n !} .
$$


and using polar coordinates we see that $\mathcal{F}_{u, v}^{\nu} \psi(\xi)$ takes the form

$$
\frac{\ell_{u, v}}{\pi} \sum_{m, n=0}^{\infty} \int_{0}^{2 \pi} \int_{0}^{\infty} \psi\left(r e^{i \theta}\right) e^{i(n-m) \theta} \frac{(u \xi)^{m}(v \bar{\xi})^{n}\left(\ell_{u, v} r\right)^{m+n}}{m ! n !} e^{-\ell_{u, v}\left(r^{2}+u v|\xi|^{2}\right)} r d r d \theta
$$

where $\ell_{u, v}$ stands for $\ell_{u, v}:=\nu /(1-u v)$. Therefore, for every rotational symmetric function $\psi_{k}(\zeta)=\Psi(|\zeta|) e^{i k \theta}$, it reduces further to

$$
2 \ell_{u, v}\left(\frac{u \xi}{v \bar{\xi}}\right)^{k / 2} \int_{0}^{\infty} r \Psi(r)\left(\sum_{m=0}^{\infty} \frac{(-i)^{2 m+k}}{m !(k+m) !}\left(i \ell_{u, v} \sqrt{u v}|\xi| r\right)^{2 m+k}\right) e^{-\ell_{u, v}\left(r^{2}+u v|\xi|^{2}\right)} d r
$$

Hence, by means of [1, p.222]

$$
I_{\alpha}(\xi):=\sum_{n=0}^{\infty} \frac{1}{n ! \Gamma(\alpha+n+1)}\left(\frac{\xi}{2}\right)^{2 n+\alpha}
$$

and $I_{\alpha}(-\xi)=(-1)^{\alpha} I_{\alpha}(\xi)$, it follows

$$
\mathcal{F}_{u, v}^{\nu} \psi_{k}(\xi)=2 \ell_{u, v}\left(\frac{u \xi}{v \bar{\xi}}\right)^{k / 2} \int_{0}^{\infty} r \Psi(r) I_{k}\left(\frac{2 \nu \sqrt{u v}}{1-u v}|\xi| r\right) e^{-\ell_{u, v}\left(r^{2}+u v|\xi|^{2}\right)} d r
$$

Proof of Theorem 1.3. Notice first that for arbitrary $f \in L_{\nu}^{2}(\mathbb{C})$, we have

$$
f\left(r e^{i \theta}\right)=\sum_{k \in \mathbb{Z}} g_{k}(r) e^{i n \theta} .
$$

Therefore, by setting $\xi=\rho e^{i \varphi}$ and making appeal of Proposition 5.1 we get

$$
\mathcal{F}_{u, v}^{\nu} f(\xi)=\sum_{k \in \mathbb{Z}}\left(\frac{\xi}{\bar{\xi}}\right)^{k / 2} \mathcal{H}_{u, v}^{\nu, \alpha}\left(g_{k}\right)(\rho)=\sum_{k \in \mathbb{Z}} \mathcal{H}_{u, v}^{\nu, \alpha}\left(g_{k}\right)(\rho) e^{i k \varphi} .
$$

Accordingly, by identification to $\mathcal{F}_{u, v}^{\nu} f\left(\rho e^{i \varphi}\right)=\sum_{k \in \mathbb{Z}} G_{k}(\rho) e^{i k \varphi}$, we see that the Fourier coefficients $G_{k}(\rho)$ and $g_{k}(r)$ of $\theta \longmapsto \mathcal{F}_{u, v}^{\nu} f\left(\rho e^{i \theta}\right)$ and $\theta \longmapsto f\left(r^{i \theta}\right)$, respectively, satisfy

$$
G_{k}(\rho)=\mathcal{H}_{u, v}^{\nu, \alpha}\left(g_{k}\right)(\rho) \text {. }
$$

\section{REFERENCES}

[1] Andrews G.E., Askey R., Roy R. Special functions. Encyclopedia of Mathematics and its Applications, 71. Cambridge University Press: Cambridge; 1999.

[2] Bargmann V., On a Hilbert space of analytic functions and an associated integral transform. Comm. Pure Appl. Math., 14 (1961) 187-214.

[3] Condon E.U., Immersion of the Fourier transform in a continuous group of functional transformations. Proc. Natl. Acad. Sci., USA 23 (1937) 158-164.

[4] Chen Y., Liu Y., On the eigenfunctions of the complex Ornstein-Uhlenbeck operators. Kyoto J. Math., vol.54, no.3 (2014) 577-596

[5] Ghanmi A., Operational formulae for the complex Hermite polynomials $H_{p, q}(z, \bar{z})$. Integral Transforms Spec. Funct., 2013; 24 (11):884-895.

[6] Ghanmi A., Mehler's formulas for the univariate complex Hermite polynomials and applications. Math. Methods Appl. Sci., 40, no. 18 (2017), 7540-7545.

[7] Ghanmi A., On dual transform of fractional Hankel transform. Preprint 2020.

[8] Hörmander L., Symplectic classification of quadratic forms, and general Mehler formulas. Mathematische Zeitschrift, 219 (1995) 413-449.

[9] Intissar A., Intissar A. Spectral properties of the Cauchy transform on $L^{2}\left(\mathbb{C} ; e^{-|z|^{2}} d \lambda\right)$. J. Math. Anal. Appl., (2006); 313 (2):400-418. 
[10] Ismail M.E.H., Analytic properties of complex Hermite polynomials. Trans. Amer. Math. Soc., 2016; 368 (2):1189-1210.

[11] Itô K., Complex multiple Wiener integral. Jap. J. Math., 1952; 22 :63-86.

[12] Kibble W.F., An extension of a theorem of Mehler's on Hermite polynomials. Proc. Cambridge Philos. Soc., 41 (1945) 12-15,

[13] Louck J. D., Extension of the Kibble-Slepian formula for Hermite polynomials using boson operator methods. Advances in Applied Mathematics, 2 (3) (1981) 239-249.

[14] Mehler F.G., Ueber die Entwicklung einer Function von beliebig vielen Variabeln nach Laplaceschen Functionen höherer Ordnung. J. Reine Angew. Math., 1866; 66:161-176.

[15] Slepian D., On the symmetrized Kronecker power of a matrix and extensions of Mehler's formula for Hermite polynomials. SIAM Journal on Mathematical Analysis, 3 (4) (1972) 606-616,

[16] Stanton D., Orthogonal polynomials and combinatorics, in Special Functions 2000: Current Perspective and future directions, Kluwer, (2000) 389-410.

[17] Srivastava H.M., Singhal J. P., Some extensions of the Mehler formula. Proc. Amer. Math. Soc., 31 (1972) 135-141.

[18] Wiener N., Hermitian Polynomials and Fourier Analysis. Journal of Mathematics and Physics 8 (1929) 70-73.

[19] Wünsche A., Transformations of Laguerre 2D-polynomials and their applications to quasiprobabilities, J. Phys. A., 1999; 21:3179-3199.

[20] Zayed A., Two-dimensional fractional Fourier transform and some of its properties. Integral Transforms Spec. Funct., 29 (7) (2018) :553-570.

E-mail address: abdelhadi.benahmadi@gmail.com

E-mail address: allalghanmi@um5.ac.ma

Analysis, P.D.E. \& Spectral Geometry, Lab M.I.A.-S.I., CeRemar, Department of Mathematics, P.O. Box 1014, Faculty of Sciences,

Mohammed V University in Rabat, Morocco 